\title{
Ab initio configuration-interaction studies of the ground state potential energy and hyperfine coupling constants of ${ }^{35} \mathrm{Cl}_{2}^{-}$
}

\author{
by S. P. KARNA $†$ and FRIEDRICH GREIN $\ddagger$ \\ Department of Chemistry, University of New Brunswick, \\ Fredericton, N. B., Canada E3B 6E2 \\ B. ENGELS and S. D. PEYERIMHOFF \\ Lehrstuhl für Theoretische Chemie, Universität Bonn, \\ Wegelerstrasse 12, D-5300 Bonn 1, F.R. Germany
}

(Received 10 July 1989; accepted 24 July 1989)

\begin{abstract}
Potential energy and spectroscopic constants for the $X^{2} \Sigma_{u}^{+}$ground state of $\mathrm{Cl}_{2}^{-}$, were calculated by configuration-interaction $(\mathrm{CI})$ methods, using large basis sets with polarization and diffuse functions. From these $\mathrm{CI}$ wavefunctions, the isotropic $\left(a_{\mathrm{iso}}\right)$ and dipolar $\left(A_{\mathrm{dip}}\right)$ components of the hyperfine coupling constant were obtained. The effects of various $s, p$ basis sets, polarization and diffuse functions, as well as the influence of reference configurations and configuration selection thresholds were investigated. The best values obtained are $35.31 \mathrm{G}$ for $a_{\text {iso }}$ and $29.44 \mathrm{G}$ for $A_{\text {dip }}$, to be compared with experimental values of $37 \pm 1 \mathrm{G}$ and $32 \pm 1 \mathrm{G}$, respectively. It is shown that the contributions to $a_{\mathrm{ino}}$ of the $K$ and $L$ shells are opposite in sign, differing by about $4 \mathrm{G}$. Upon vibrational averaging, both $a_{\text {iso }}$ and $A_{\text {dip }}$ move towards smaller values as $v$ increases. An adiabatic electron affinity of $2.46 \mathrm{eV}$ was obtained for $\mathrm{Cl}_{2}$, and a vertical electron detachment energy of $3.71 \mathrm{eV}$ for $\mathrm{Cl}_{2}^{-}$.
\end{abstract}

\section{Introduction}

Electron spin resonance (E.S.R.) data on the hyperfine coupling constants (hfcc) of the $\mathrm{F}_{2}^{-}$and $\mathrm{Cl}_{2}^{-}$anions have been available for some time [1-4]. Several ab initio quantum mechanical investigations on the hfcc of $F_{2}^{-}[5-9]$ and other first-row systems [10-17] have recently appeared in the literature. From such studies, the importance of the choice of basis set in finite basis calculations, of spin polarization contributions from the inner shell electrons which are normally neglected in frozen core configuration-interaction (CI) calculations, and of the molecular geometry has been amply demonstrated.

In this paper ab initio $\mathrm{CI}$ results on $\mathrm{Cl}_{2}^{-}$, the second-row homologue of $\mathrm{F}_{2}^{-}$, will be reported. While second-row systems are generally expected to be more difficult to handle, due to the larger number of electrons, there are indications that the hfec of $\mathrm{Cl}_{2}^{-}$is easier to obtain than that of $\mathrm{F}_{2}^{-}$. For example, the unrestricted Hartree-Fock (UHF) method consistently overestimates the isotropic hfcc of $F_{2}^{-}$, while the same method gives results for $\mathrm{Cl}_{2}^{-}$which are within the range of the experimental values

† Present address: IBM Corporation, Department 48B/MS428, Kingston, New York 12401, U.S.A.

$\ddagger$ Author to whom all correspondence should be sent. 
[8]. Whereas in UHF calculations $d$ functions and contraction schemes for the basis functions strongly influence the calculated hfccs of $F_{2}^{-}$, such effects in the case of $\mathrm{Cl}_{2}^{-}$have been noted to be small [8].

It is known that for $F_{2}^{-}[6,9]$ and other first-row systems [13-15], the spin polarization contributions due to the core $(n=1)$ electrons are similar in magnitude but opposite in sign to the corresponding contributions from the valence electrons $(n=2)$. Since the total spin density is the sum of such various contributions, neglect of either core or valence spin polarization contributions is expected to give poor results. Therefore, for first-row systems a frozen core CI treatment is bound to fail. However, on addition of another shell, CI treatments for the valence shell, with frozen $K$ and $L$ shells, can benefit from a partial cancellation of the spin polarization contributions of the $K$ and $L$ shells. If one is forced to perform frozen core CI calculations, due to space restrictions, then it is important to know the size of the error introduced in the hfcc due to neglecting the contributions from the inner shell electrons.

The experimental geometry of $\mathrm{Cl}_{2}^{-}$is not available. The UHF/PUHF (projected UHF) calculations reported in reference [5] were performed at the restricted Hartree-Fock (RHF) optimized distance of $5.0 a_{0}$ [18]. In the present study we calculated the $\mathrm{CI}$ potential energy curve and the corresponding equilibrium constants for the $X^{2} \Sigma_{w}^{+}$state of $\mathrm{Cl}_{2}^{-}$. While the final hfcc's were calculated at the $\mathrm{CI}$ equilibrium geometry, for comparison with the previous PUHF results the effect of basis sets and $\mathrm{Cl}$ parameters were studied at $R=5.0 a_{0}$. This is not expected to affect the generality of the results, since the two geometries differ by less than $0.04 a_{0}$.

\section{Theoretical treatment}

The equations used for calculating the isotropic, $a_{\text {iso }}$, and dipolar, $A_{\text {dip }}$, parts of the hyperfine coupling constant were given in $[8,9]$. The CI wavefunctions were obtained from the multireference single and double excitation (MRD)-CI method of Buenker and Peyerimhoff [18-21]. The special integrals needed for the hyperfine coupling calculations were obtained according to Chandra and Buenker [22].

Initially, the potential energy of the $X^{2} \Sigma_{u}^{+}$state of $\mathrm{Cl}_{2}^{-}$between $R=4.0$ and 6.0 $a_{0}$ was calculated, using a (12s8p/6s4p) contracted gaussian basis set for $\mathrm{Cl}^{-}$[23] (later called basis $D$ ), augmented by two $d$ and an $f$ polarization function [24] and $s$, $p$ diffuse functions [25]. Molecular orbitals (MO) obtained for the $X^{2} \Sigma_{u}^{+}$state of $\mathrm{Cl}_{2}^{-}$were employed in the $\mathrm{CI}$ calculations. MOs corresponding to $n=1$ and 2 at each centre were kept doubly occupied (frozen) while the corresponding 10 highestenergy MOs were not populated (discarded). The CI wavefunction consisted of all single and double excitations of 15 valence electrons over a space of $48 \mathrm{MOs}$.

Initially, four reference configurations (mains: $M$ ), namely

(i) (core) $4 \sigma_{g}^{2} 4 \sigma_{u}^{2} 2 \pi_{u}^{4} 2 \pi_{g}^{4} 5 \sigma_{\theta}^{2} 5 \sigma_{u}(0 \cdot 91)$,

(ii) (core) $4 \sigma_{g}^{2} 4 \sigma_{\varepsilon}^{2} 2 \pi_{u}^{4} 2 \pi_{g}^{4} 5 \sigma_{\theta} 5 \sigma_{u} 7 \sigma_{g}(0.0024)$,

(iii) and (iv) (core) $4 \sigma_{\mathrm{g}}^{2} 4 \sigma_{u}^{2} 2 \pi_{u}^{4} 2 \pi_{\sigma}^{3} 5 \sigma_{\sigma} 5 \sigma_{u}{ }^{2} 3 \pi_{u}(0.00016)$,

where the core is $1 \sigma_{g}^{2} 1 \sigma_{u}^{2} 2 \sigma_{g}^{2} 2 \sigma_{u}^{2} 3 \sigma_{g}^{2} 3 \sigma_{u}^{2} 1 \pi_{u}^{4} 1 \pi_{g}^{4}$ were employed, with a configuration selection threshold $T=10.0 \mu \mathrm{h}$. The selection of the mains was made on the basis of their contributions $\left(C^{2}\right)$ to the total $\mathrm{CI}$ wave function. The numbers in parentheses indicate the $C^{2}$ value for the corresponding reference configuration at $5 a_{0}$. Subsequently, the reference configurations (iii) and (iv) were excluded from the 
Table 1. Exponents of $d, f$ and diffuse functions.

\begin{tabular}{lcc}
\hline Function & Exponent & Reference \\
\hline $1 d$ & 0.60 & 23 \\
$2 d$ & 1.05 & 23 \\
& 0.35 & \\
$3 d$ & 2.12 & 27 \\
& 0.76 & \\
$1 f$ & 0.27 & \\
$p_{\text {neg }}$ & 0.75 & 23 \\
$s_{\text {diff }}$ & 0.049 & 24 \\
$p_{\text {diff }}$ & 0.025 & 24 \\
\hline
\end{tabular}

final calculation of the potential function which was carried out at a selection threshold of $5.0 \mu \mathrm{h}$.

For the calculation of the hfccs, four different $s, p$ basis sets were tested. The first set, called $A$, consisted of the chlorine 12s7p gaussian functions of Huzinaga [26] in the $6 s 4 p$ contraction of Dunning and Hay [25], with the coefficients corrected according to Craven $e$ al. [27]. The second basis set $(B)$ was constructed by adding $p_{\text {neg }}$ functions [25] to basis $A$. The third $(C)$ and fourth $(D)$ basis sets were the $12 s 8 p$ gaussian functions in a $6 s 4 p[23,26]$ contraction for the $\mathrm{Cl}$ atom and $\mathrm{Cl}^{-}$ion, respectively. Subsequently one, two and three $d$ functions $[24,28]$, an $f$ function [24] and diffuse $s$ and $p$ functions [25] were added to basis set $D$. The exponents of the additional functions along with the corresponding references are listed in table 1.

To study the spin polarization contributions from various occupied MOs, basis set $D$ plus $2 d 1 f$ and diffuse $s$ and $p$ functions (same as used for the potential energy calculation) was employed using one reference configuration and a configuration selection threshold of $5 \mu \mathrm{h}$. Initially, all but the $1 \pi_{u}^{4} 1 \pi_{g}^{4}$ electrons were correlated. This left 27 electrons to be correlated, and the corresponding result is taken as the 'all-electron' value. In subsequent calculations the MO pairs $\left(1 s \sigma_{g}, 1 s \sigma_{v}\right),\left(2 s \sigma_{g}\right.$, $\left.2 s \sigma_{u}\right),\left(2 p_{z} \sigma_{g}, 2 p_{z} \sigma_{u}\right)$ and $\left(3 s \sigma_{g}, 3 s \sigma_{u}\right)$ were kept doubly occupied one at a time. The individual contributions to the spin density were obtained from the difference between the 27-electron and the 23-electron calculations for a given MO pair. In such studies the inter-orbital correlation is not incorporated, and therefore only an estimate of the spin polarization contribution to the total spin density is obtained.

\section{Results and discussion}

\subsection{Spectroscopic constants}

Equilibrium spectroscopic constants for the $\mathrm{Cl}_{2}^{-}$radical anion in the gas phase are not available in the literature. However, several experimental measurements of the vibrational frequencies of $\mathrm{Cl}_{2}^{-}$trapped in solid matrices [29] and generated in solutions by radiolysis of chloride ions [30-32] have been made. Although the corresponding values of $\omega_{\mathrm{e}}$ and $\omega_{\mathrm{e}} x_{\mathrm{e}}$ are expected to differ from the corresponding gas phase values, they provide a useful guide for comparison with our theoretical results. The potential energies for the $X^{2} \Sigma_{u}^{+}$state of $\mathrm{Cl}_{2}^{-}$obtained in the present 
Table 2. Potential energy and hyperfine coupling constants of the $X^{2} \Sigma_{u}^{+}$state of $\mathrm{Cl}_{2}^{-}$as function of $R \dagger$.

\begin{tabular}{cccc}
\hline$R / \mathrm{a}_{0}$ & $E_{\mathrm{C}} / \mathrm{h}$ & $a_{\mathrm{iso}} / \mathrm{G}$ & $A_{\mathrm{dip}} / \mathrm{G}$ \\
\hline 4.0 & -919.37282 & 96.72 & 25.47 \\
4.2 & -919.38937 & 80.32 & 26.82 \\
4.4 & -919.40049 & 66.60 & 27.60 \\
4.6 & -919.40625 & 55.32 & 27.97 \\
4.8 & -919.40904 & 46.20 & 28.10 \\
5.0 & -919.40966 & 38.90 & 28.10 \\
5.2 & -919.40845 & 33.19 & 28.04 \\
5.4 & -919.40609 & 28.49 & 27.94 \\
5.6 & -919.40312 & 23.83 & 27.80 \\
5.8 & -919.39953 & 21.85 & 27.65 \\
6.0 & -919.39521 & 19.25 & 27.49 \\
\hline
\end{tabular}

$\dagger$ Basis $D+2 d+1 f \overline{+(s, p)_{\text {diffuse }} \cdot 2 M / 5 \mu \text { h calculations. } 1 a_{0} \approx 5 \cdot 2918} \times 10^{-11} \mathrm{~m}, 1 E_{\mathrm{h}} \approx$ $4.359 \times 10^{-18} \mathrm{~J}, 1 \mathrm{G}=10^{-4} \mathrm{~T}$.

study are listed in table 2 . The corresponding equilibrium constants along with some experimental counterparts are listed in table 3.

The values of $\omega_{e}$ and $\omega_{e} x_{e}$ obtained in the present study show good agreement with the experimental values, especially with those of Tripathi $e t$ al. [31] and Hynes et al. [32]. The present calculation lends support to the smaller value of the anharmonic constant $\omega_{\mathrm{e}} x_{\mathrm{e}}\left(1.43-1.83 \mathrm{~cm}^{-1}\right)$ obtained by Tripathi et al. [31] and Hynes et al. [32] rather than the value of $3.83 \mathrm{~cm}^{-1}$ obtained by Wilbrandt et al. [30]. This observation is important, especially in resolving the recent controversy [30-32] regarding the dissociation energy of $\mathrm{Cl}_{2}^{-}$. Based upon the larger value of $\omega_{\mathrm{e}} x_{\mathrm{e}}$, Wilbrandt et al. [30] estimated the dissociation energy of $\mathrm{Cl}_{2}^{-}$to be $0.66 \mathrm{eV}$, which is substantially smaller than the value of 1.26 to $1.6 \mathrm{eV}$ obtained from thermochemical data [33] and from spectroscopic methods in solid matrices [29] and in aqueous solution [31,32]. Unfortunately, due to difficulties encountered in the SCF convergence at larger internuclear distances, we could not obtain the dissociation

Table 3. Spectroscopic constants and hyperfine coupling constants of the $X^{2} \Sigma_{u}^{+}$state of

$$
{ }^{35} \mathrm{Cl}_{2}^{-\dagger} \text {. }
$$

\begin{tabular}{lcc}
\hline & This work & Experiment \\
\hline$E_{\min } / \mathrm{h}$ & -919.40970 & \\
$R_{\mathrm{e}}$ & $2.6274 \AA\left(4.965 a_{0}\right)$ & \\
$\omega_{\mathrm{e}} / \mathrm{cm}^{-1}$ & 274.87 & $277.0 \pm 0.38$ \\
$\omega_{\mathrm{e}} x_{\mathrm{e}} / \mathrm{cm}^{-1}$ & 1.71 & $1.83 \pm 0.05 \S$ \\
$B_{e} / \mathrm{cm}^{-1}$ & 0.1397 & \\
$\rho^{3} / \mathrm{a} . \mathrm{u}$ & $0.24650(0.22571) \ddagger$ & \\
$a_{\mathrm{led}} / \mathrm{G}$ & $38.56(35.31) \ddagger$ & $37 \pm 1 \|$ \\
$A_{\mathrm{dip}} / \mathrm{G}$ & $28.21(29.44) \ddagger$ & $32 \pm 1 \|$ \\
\hline
\end{tabular}

† Theoretical values of hfocs obtained at the calculated $R_{e}$, with 15 electrons correlated. $\ddagger$ Values in parentheses obtained from 27 electron calculations (see text).

$\S[31]$, see text.

|| [2]. 
energy of $\mathrm{Cl}_{2}^{-}$. However, our results for $\omega_{\mathrm{e}}$ and $\omega_{\mathrm{e}} x_{\mathrm{e}}$ support a value of $D_{\mathrm{e}} \simeq 1 \cdot 3 \mathrm{eV}$, in agreement with Hartree-Fock (HF) calculations of Gilbert and Wahl [18].

The CI value of $R_{e}\left(4.965 a_{0}\right.$, table 3) compares well with a HF value of $5 a_{0}$ [18]. Due to the small difference in these two $R_{\mathrm{c}}$ values, the hfccs calculated at $5 a_{0}$ hardly show any difference from those obtained at $4.965 a_{0}$.

We also calculated the electron affinity $(\mathrm{EA})$ of $\mathrm{Cl}_{2}$. Although not a major concern in the context of the present study, the calculation of EA provides a measure of the ability of the wavefunction to stabilize the extra electron, which is important in the calculation of hfccs of negative ions. For the calculation of EA, the experimental value of $R_{\mathrm{e}}=3.755 a_{0}$ for the $X^{1} \Sigma_{g}^{+}$state of $\mathrm{Cl}_{2}$ [33] and the calculated value of $R_{\mathrm{c}}=4.965 \mathrm{a}_{0}$ for $X^{2} \Sigma_{u}^{+}$of $\mathrm{Cl}_{2}^{-}$were used. The vertical detachment energy was calculated at $R_{\mathrm{e}}\left(\mathrm{Cl}_{2}^{-}\right)$. MOs obtained for the $X^{2} \Sigma_{\mathrm{u}}^{+}$state $\mathrm{Cl}_{2}^{-}$were employed for both the neutral molecule and the anion radical, using the basis set of table 2. For $\mathrm{Cl}_{2}, 6$ mains were chosen and 2 mains for $\mathrm{Cl}_{2}^{-}$. In both cases, the selection threshold was $2 \mu \mathrm{h}$. An adiabatic electron affinity for $\mathrm{Cl}_{2}$ of $2.46 \mathrm{eV}$ was obtained. This value can be compared with the corresponding thermochemical value of $2.39 \mathrm{eV}$ [33] and an electron attachment value of $2.52 \mathrm{eV}$ [33]. The calculated vertical detachment energy of $\mathrm{Cl}_{2}^{-}$is $3 \cdot 7 \mathrm{eV}$.

\subsection{Hyperfine coupling constants}

In the previous section, $\mathrm{CI}$ studies were described for obtaining potential curves and spectroscopic constants. Values for $a_{\text {iso }}$ and $A_{\text {dip }}$, for each value of $R$, are given in table 2. Values at the $\mathrm{Cl}$ optimized value of $R$ are shown in table 3 . It should be noted that in these calculations $K$ and $L$ electrons were frozen. Estimates of results for 27 valence electrons, to be discussed later, are given in parentheses in table 3.

Results obtained with the four basis sets $A$ to $D$ using one (1M) or four (4M) reference configurations with frozen core and configuration selection thresholds of 0 or $5 \mu \mathrm{h}$ are listed in table 4 . The contribution of the reference configurations to the CI wave function is 95 to 96 per cent.

As far as the hfccs are concerned, the four basis sets in table 4 give similar results. The $a_{\text {iso }}$ values differ by less than 1 per cent, and the largest changes

Table 4. Hyperfine coupling constants obtained for $X^{2} \Sigma_{u}^{+}$state of $\mathrm{Cl}_{2}^{-}$, using various basis sets, numbers of reference configurations $(M)$ and selection thresholds. $†$

\begin{tabular}{ccccc}
\hline Basis set & $\mathrm{CI}$ & $E / \mathrm{h}$ & $a_{\text {iso }} / \mathrm{G}$ & $A_{\text {dip }} / \mathrm{G}$ \\
\hline$A$ & $1 \mathrm{M} / 0 \mu \mathrm{h}$ & -919.06756 & 39.53 & 28.45 \\
$B$ & $1 \mathrm{M} / 0 \mu \mathrm{h}$ & -919.09842 & $39 \cdot 10$ & 27.66 \\
$C$ & $1 \mathrm{M} / 0 \mu \mathrm{h}$ & -919.12442 & 39.85 & 28.83 \\
& $4 \mathrm{M} / 5 \mu \mathrm{h}$ & $-919 \cdot 12501$ & 40.12 & 28.87 \\
& $4 \mathrm{M} / 0 \mu \mathrm{h}$ & $-919 \cdot 12629$ & 40.61 & 29.08 \\
$D$ & $1 \mathrm{M} / 0 \mu \mathrm{h}$ & -919.12916 & 39.93 & 28.28 \\
& $4 \mathrm{M} / 5 \mu \mathrm{h}$ & -919.13002 & 40.16 & 28.33 \\
& $4 \mathrm{M} / 0 \mu \mathrm{h}$ & -919.13164 & 40.61 & 28.55 \\
\hline
\end{tabular}

† At $R=5 a_{0}$. 
observed for $A_{\text {dip }}$ are 3 to 4 per cent. Considering the energies, basis $C$ and $D$ are superior to basis sets $A$ and $B$, with basis $D\left(\mathrm{Cl}^{-}\right)$being the best. For $4 M$ calculations, $a_{\text {iso }}$ increased by less than 2 per cent from its $1 M$ counterpart, whereas $A_{\text {dip }}$ hardly changes.

Results obtained by using basis $D$ with and without polarization and diffuse functions are listed in table 5. Also listed are the PUHF values for comparison. The addition of one $d$ function to the $6 s 4 p$ basis set results in a decrease of about 9 per cent in the value of $a_{\mathrm{iso}}$, smaller than the corresponding change for $\mathrm{F}_{2}^{-}(\sim 24 \mathrm{per}$ cent) [9]. Such a change at the CI level can be understood in terms of a larger correlating orbital space for the valence electrons of $\mathrm{Cl}_{2}^{-}$. The mechanism for spin polarization at the UHF level is different. There, the $d$ functions help improve the spin density at the nucleus largely via their $s\left(x^{2}+y^{2}+z^{2}\right)$ component and the corresponding change at the PUHF level is very small. Further addition of $d$ functions does not affect the hfcc greatly, and the trend in the CI results is parallel to the PUHF results [8]. The small change of $a_{\text {iso }}$ due to $d$ functions may also be related to correlating only the valence electrons in the present case.

The $f$ function does not seem to influence the spin density of $\mathrm{Cl}_{2}^{-}$. This behaviour is not unusual since the configurations resulting from $f$ functions require double excitations. The spin polarization contributions from such excitations are fairly small. As in other situations, $A_{\text {dip }}$ is not affected by $d$ and $f$ functions.

\subsection{Analysis of spin polarization contributions from various shells}

It was noted in the previous sections that the results obtained for $a_{\text {iso }}$ from the frozen core $\mathrm{Cl}$ calculations were close to the experimental value of $37 \mathrm{G}$. With the best basis set of table $5, a_{\text {iso }}$ is $35.64 \mathrm{G}$. The $7 s 5 p 2 d 1 f$ basis set of table 2, differing from $6 s 4 p 2 d 1 f$ of table 5 by the addition of diffuse $s$ and $p$ functions, gave $38.90 \mathrm{G}$ at $R=5 a_{0}$, using 2 mains and $T=5 \mu \mathrm{h}$.

Since the core of $\mathrm{Cl}_{2}^{-}$contains the $\sigma$ orbital pairs $\left(1 s \sigma_{g}, 1 s \sigma_{u}\right)$ for $n=1$ and $\left(2 s \sigma_{g}\right.$, $\left.2 s \sigma_{u}\right)$ and $\left(2 p \sigma_{q}, 2 p \sigma_{u}\right)$ for $n=2$ it is possible that the spin polarization of the $n=1$ electrons is similar in magnitude but opposite in sign to that of the $n=2$ electrons. In that case, the frozen core approximation would lead to (accidentally) good results

Table 5. Effect of polarization and diffuse functions on hfocs of ${ }^{35} \mathrm{Cl}_{2}^{-} \cdot+$

\begin{tabular}{|c|c|c|c|c|c|c|}
\hline \multirow[b]{2}{*}{ Basis $\ddagger$} & \multirow[b]{2}{*}{ CI } & \multirow[b]{2}{*}{$E_{\mathrm{c}} / \mathbf{h}$} & \multicolumn{2}{|c|}{$a_{\text {iboo }} / \mathbf{G}$} & \multicolumn{2}{|c|}{$A_{d i p} / \mathrm{G}$} \\
\hline & & & CI & PUHF\& & CI & PUHF \\
\hline $\begin{array}{l}\text { 6s4p } \\
7 s 5 p \| \\
6 s 4 p 1 d \\
6 s 4 p 2 d \\
6 s 4 p 3 d \\
6 . s 4 p 2 d 1 f\end{array}$ & $\begin{array}{l}1 \mathrm{M} / 0 \mu \mathrm{h} \\
1 \mathrm{M} / 0 \mu \mathrm{h} \\
1 \mathrm{M} / 0 \mu \mathrm{h} \\
1 \mathrm{M} / 0 \mu \mathrm{h} \\
1 \mathrm{M} / 0.05 \mu \mathrm{h} \\
1 \mathrm{M} / 0.5 \mu \mathrm{h}\end{array}$ & $\begin{array}{l}-919 \cdot 12916 \\
-919 \cdot 13351 \\
-919 \cdot 30963 \\
-919 \cdot 34651 \\
-919 \cdot 35280 \\
-919 \cdot 40892\end{array}$ & $\begin{array}{l}39 \cdot 93 \\
40 \cdot 05 \\
36 \cdot 21 \\
37.00 \\
36 \cdot 22 \\
35 \cdot 64\end{array}$ & $\begin{array}{l}34 \cdot 7 \\
34 \cdot 5 \\
33 \cdot 2 \\
34 \cdot 0 \\
32 \cdot 6 \\
32 \cdot 6\end{array}$ & $\begin{array}{l}28.28 \\
27.98 \\
27.89 \\
27.83 \\
27.82 \\
28.28\end{array}$ & $\begin{array}{l}28 \cdot 6 \\
28.1 \\
28 \cdot 6 \\
28 \cdot 5 \\
28 \cdot 6 \\
28 \cdot 7\end{array}$ \\
\hline
\end{tabular}

† At $R=5 \cdot 0 a_{0}$.

$\mp 6 s 4 p$ is basis $D$, see text.

$\S$ Projected unrestricted Hartree-Fock results from [8]. The $s, p$ basis set was basis $A$.

Polarization and diffuse functions the same as here.

$\| 7 s 5$ is $6 s 4 p+(s, p)_{\text {diffuse }}$. 
Table 6. Contributions of $\sigma_{g}$ and $\sigma_{\mathrm{w}} \mathrm{MOs}$ to spin density, $\rho^{2}$, and $a_{\mathrm{iso}}$, for ${ }^{35} \mathrm{Cl}_{2}^{-} \dagger$.

\begin{tabular}{lrr}
\hline & \multicolumn{1}{c}{$\rho^{z} /$ a.u. } & \multicolumn{1}{c}{$a_{\text {ise }} / \mathrm{G}$} \\
\hline $1 s \sigma_{g}^{2} 1 s \sigma_{u}^{2}$ & -0.06115 & -9.56 \\
$2 s \sigma_{q}^{2} 2 s \sigma_{u}^{2}$ & 0.03639 & 5.70 \\
$2 p \sigma_{s}^{2} 2 p \sigma_{u}^{2}$ & -0.00106 & -0.16 \\
$3 s \sigma_{s}^{2} 3 s \sigma_{u}^{2}$ & 0.09489 & 14.85 \\
$3 p \sigma_{v}^{2} 3 p \sigma_{u} \ddagger$ & 015008 & 23.46 \\
Total & 0.21915 & 34.29 \\
\hline
\end{tabular}

$\dagger$ See text for detail. All calculations at $5 a_{0}$.

$\ddagger$ Obtained indirectly from difference.

$\S$ 'All-electron' result.

for $a_{\text {iso }}$. To examine this assumption quantitatively, the contributions to the spin density $\rho^{z}$ and $a_{\mathrm{iso}}$ from each pair of MOs mentioned above, as well as from (3s $\sigma_{\theta}$, $3 s \sigma_{w}$ ), were calculated according to the method described in $\S 2$. The results are given in table 6. The contributions from the $3 p \sigma$ electrons, also included in table 6, were obtained as the difference between the 'all-electron' results and the sum of the inner $\sigma$ contributions.

It is noted from table 6 that the spin polarization contribution of the $1 s \sigma_{g}^{2} 1 s \sigma_{u}^{2}$ electrons is indeed opposite in sign to that of the $2 s \sigma_{g}^{2} 2 s \sigma_{u}^{2}$ electrons, with a difference of about $4 \mathrm{G}$ in the value of $a_{\text {iso }}$. This causes $a_{\text {iso }}$ to drop from $38.9 \mathrm{G}$ to $34.3 \mathrm{G}$ for the same basis set and distance. The good agreement of the frozen core CI results with experiment is thus due to a fortuitous cancellation of the $n=1$ and $n=2$ contributions. Although the error introduced by frozen core calculations can often be smaller than for first-row elements, care must be exercised in using this method for calculating the spin density in second-row systems. As table 3 shows, 'all-electron' CI wavefunctions increase $A_{\text {dip }}$ by about $1 \cdot 2 \mathrm{G}$. Due to the small changes in $A_{\text {dip }}$, an analysis of contributions from various shells has not been attempted.

Since the hfcc of $\mathrm{Cl}_{2}^{-}$, especially the isotropic part, is very sensitive to the intermolecular distance (table 2), it is of interest to obtain $a_{\text {iso }}$ and $A_{\text {dip }}$ for the low-lying vibrational levels of the $X^{2} \Sigma_{u}^{+}$state. Using the potential energy curves, expectation values $\left\langle v\left|a_{\text {iso }}\right| v\right\rangle$ and $\left\langle v\left|A_{\text {dip }}\right| v\right\rangle$ along with $\varepsilon_{v}$ for $v=0$ to 4 have been obtained. They are listed in table 7. Corresponding experimental data are not available. The calculated value of $a_{\mathrm{iso}}(v=0)$, corrected for the frozen core error, is within

Table 7. Hyperfine coupling constants of $\mathrm{Cl}_{2}^{-}$averaged over five lowest vibrational levels $\dagger$.

\begin{tabular}{crcc}
\hline$v$ & $\varepsilon_{\mathrm{v}} / \mathrm{cm}^{-1}$ & $a_{\text {iso }} / \mathrm{G}$ & $A_{\text {dip }} / \mathrm{G}$ \\
\hline 0 & 137.01 & $40.36(35.75)$ & $28.09(29.29)$ \\
1 & 408.47 & $40.31(35.70)$ & $28.08(29.27)$ \\
2 & 676.54 & $40.02(35.40)$ & $28.04(29.23)$ \\
3 & 941.21 & $39.57(34.95)$ & $28.01(29.21)$ \\
4 & 1202.47 & $38.98(34.37)$ & $27.99(29.18)$ \\
\hline
\end{tabular}

† Basis set and $\mathrm{CI}$ as in table 2.

$\ddagger$ Values in parentheses have been corrected for the frozen core electrons. 
3 per cent of the corresponding Ar-matrix value of $37 \mathrm{G} \mathrm{[2]} \mathrm{(table} \mathrm{3),} \mathrm{while} \mathrm{a} \mathrm{larger}$ disagreement ( $\sim 8$ per cent) is noted for $A_{\text {dip }}$.

\section{Summary and conclusion}

Previous UHF studies showed that $a b$ initio hyperfine coupling constants of ${ }^{35} \mathrm{Cl}_{2}^{-}$are fairly independent of the choice of the $s, p$ basis set, and change by only small amounts when $d$ and $f$ functions are added. The $\mathrm{CI}$ results reported in this paper confirm such earlier observations. Several contracted gaussian basis sets taken from the literature give virtually identical results for the hfccs. Upon addition of the first $d$ function, $a_{\text {iso }}$ drops from 39.93 to $36.21 \mathrm{G}$ (table 5), a much smaller relative drop than seen for the corresponding first-row system ${ }^{19} \mathrm{~F}_{2}^{-}$. Further $d$ functions accomplish no change, whereas one additional $f$ function lowers $a_{\text {iso }}$ by $0.6 \mathrm{G}$. Diffuse functions have a tendency to increase $a_{\text {iso }}$ slightly. As observed for other systems, $A_{\text {dip }}$ remains unchanged.

Due to dimensional limitations in the computer programs, not all electrons could be correlated. However, it was possible to correlate the $1 s$ and $2 s$ electrons, along with all valence electrons, freezing only the $2 p \pi$ portion which is not expected to make a significant contribution to the hfccs. The separate contributions from the $1 s, 2 s, 2 p \sigma$ and $3 s$ electrons to $a_{\text {iso }}$ were obtained. The 'all-electron' values for $a_{\text {iso }}$ are 3 to $4 \mathrm{G}$ lower than the frozen-core values. Analysis shows that the $1 s$ contributions to $a_{\mathrm{isb}}$ are $-9.5 \mathrm{G}$, whereas the $2 s$ and $2 p \sigma$ contributions are $+5.5 \mathrm{G}$, explaining the above difference. Frozen core $\mathrm{CI}$ calculations for second-row systems may lead to reasonable results for $a_{\text {iso }}$ due to partial cancellation of $1 s$ and $2 s, 2 p$ contributions. Since for first-row systems the $1 s$ contributions are also of opposite sign to the $2 s, 2 p$ contributions, frozen-core calculations for such systems are expected to lead to a larger error since the neglected $1 s$ contributions cannot be compensated for.

The isotropic hfcc shows a strong dependence on the bond distance, and the values of $a_{\text {lao }}$ for increasing vibrational levels decrease significantly.

The best calculated values of $a_{\text {iso }}$ and $A_{\text {dip }}$ are slightly lower than the corresponding experimental values. However, due to the closeness of the results, no conclusions about possible deficiencies of the theoretical methods can be drawn.

Theoretical CI studies on spectroscopic constants of the $X^{2} \Sigma_{u}^{+}$ground state of $\mathrm{Cl}_{2}^{-}$have not been reported previously. As far as comparisons can be made, there is good agreement with experimental results. The same applies to calculated electron affinities of $\mathrm{Cl}_{2}$.

We thank NSERC (Canada) for financial support through operating grants, NATO for a travel grant, and the Computing Services of this University for providing excellent computing facilities.

\section{References}

[1] SChoemaker, D., 1973, Phys. Rev. B, 7, 786.

[2] De Pinillos, J. V. M., and Weltner, W., JR., 1976, J. chem. Phys., 65, 4256.

[3] Marshall, S. A., 1977, Phys. Stat. Sol. B, 82, 311.

[4] KNight, L. B., JR., Earl, E., Ligon, A. R., and Cobranch, D. P., 1986, J. chem. Phys., $85,1228$.

[5] Nguen, M. T., and HA, T.-K., 1987, J. phys. Chem., 91, 1703. 
[6] Carmichael, I., 1987, J. phys. Chem., 91, 6443.

[7] Richman, K. W., and McCullough, E. A., JR., 1988, J. phys. Chem., 92, 2714.

[8] Karna, S. P., and Grein, F., 1989, Int. J. quant. Chem., 36, 265.

[9] Karna, S. P., Grein, F., Engels, B., and PeyerimhofF, S. D., 1989, Int. J. quant. Chem., $36,255$.

[10] ChIPMAN, D. M., 1979, J. chem. Phys., 71, 761.

[11] Karna, S. P., 1983, Ph.D. Thesis, Banaras Hindu University.

[12] Chipman, D. M., 1983, J. chem. Phys., 78, 3112.

[13] Feller, D., and DAvidson, E. R., 1984, J. chem. Phys., 80, 1006.

[14] ENGELS, B., 1987, Ph.D. Thesis, University of Bonn.

[15] Engels, B., Peyerimhofr, S. D., and Davidson, E. R., 1987, Molec. Phys., 62, 109.

[16] Feller, D., and Davidson, E. R., 1988, J. chem. Phys., 88, 7580.

[17] Carmichael, I., 1989, J. phys. Chem., 93, 190.

[18] GilBERT, T. L., and WAHL, A. C., 1971, J. chem. Phys., 55, 524.

[19] Butenker, R. J., and PeYerimhofF, S. D., 1974, Theor. chim. Acta, 35, 33; 1975, Ibid., 39, 217.

[20] BuenKer, R. J., PeYerimhoff, S. D., and Butscher, W., 1978, Molec. Phys., 35, 771.

[21] BUENKER, R. J., 1986, Int. J. quant. Chem., 29, 435, and references therein.

[22] Chandra, P., and BuenkeR, R. J., 1983, J. chem. Phys., 79, 358.

[23] MCLean, A. D., and Chandler, G. S., 1980, J. chem. Phys., 72, 5639.

[24] AhLrichs, R., KeIL, F., LischKa, H., KutzelniGg, W., and Staemmler, V., 1975, J. chem. Phys., 63, 455.

[25] Dunning, T. H., JR., and HAY, P. J., 1977, Modern Theoretical Chemistry, Methods of Electronic Structure Theory, Vol. 3, edited by H. F. Schaefer III (Plenum Press).

[26] Huzinaga, S., 1971, Approximate atomic functions, II, Department of Chemistry Report, University of Alberta, Edmonton, Alberta, Canada.

[27] Craven, W., Knowles, D. B., Murrell, J. N., Vincent, M. A., and Watts, J. D., 1985, Chem. Phys. Lett., 116, 119.

[28] BeCHERER, R., and Ahlrichs, R., 1985, Chem. Phys., 99, 389.

[29] HoWARD, W. F., JR., and ANDREWS, L., 1975, Inorg. Chem., 14, 767.

[30] Wilbrandt, R., Jensen, N.-H., Sillensen, A. H., and Hansen, K. B., 1984, Chem. Phys. Lett., 106, 503.

[31] Tripath, G. N. R., Schular, R. H., and Fessenden, R. W., 1985, Chem. Phys. Lett., $113,563$.

[32] HyNes, A. J., and Wine, P. H., 1988, J. chem. Phys., 89, 3565.

[33] HUBer, K. P., and HerzberG, G., 1979, Molecular Spectra and Molecular Structure. Vol. IV. Constants of Diatomic Molecules (Van Nostrand Reinhold). 